\title{
On the acoustic model of lithosphere-atmosphere-ionosphere coupling before earthquakes
}

\author{
C.-V. Meister ${ }^{1}$, B. Mayer $^{1}$, P. Dziendziel ${ }^{1}$, F. Fülbert ${ }^{1}$, D. H. H. Hoffmann ${ }^{1}$, and V. A. Liperovsky ${ }^{2}$ \\ ${ }^{1}$ Institut für Kernphysik, Technische Universität Darmstadt, Schlossgartenstr. 9, 64289 Darmstadt, Germany \\ ${ }^{2}$ Institute of Physics of the Earth of the Russian Academy of Sciences, Bolshaya Gruzinskaya 10, 123995 Moscow, Russia
}

Received: 16 November 2010 - Revised: 16 February 2011 - Accepted: 2 March 2011 - Published: 1 April 2011

\begin{abstract}
In this work, the many-fluid magnetohydrodynamic theory is applied to describe the modification of the electromagnetic field of the ionospheric E-layer by acoustictype waves. There, altitudinal profiles of the electromagnetic field and the plasma parameters of the atmosphere and ionosphere are taken into account. It is concluded that at Eregion altitudes above seismo-active regions, magnetohydrodynamic waves as Alfvén and magnetoacoustic ones might change their amplitude and direction of propagation. Waves of the Farley-Buneman type might also be excited a few days before very strong earthquakes. The collisions between the neutral and charged particles of the E-layer also cause diffusion and heating processes. Thus, changes of the characteristic foE-frequency might be obtained.
\end{abstract}

\section{Introduction}

Acoustic and acoustic-gravity waves were indeed observed at subionospheric altitudes during earthquake preparation times (Cook, 1971; Koshevaya et al., 2002; Rozhnoi et al., 2007; Pulinets and Boyarchuk, 2004; Hayakawa, 2011). Thus, in some of the models of lithosphere-atmosphere-ionosphere coupling before earthquakes, it is assumed that atmospheric acoustic and acoustic-gravity waves are generated several days before earthquakes in earthquake preparation zones and that they propagate from the Earth's surface through the atmosphere up to ionospheric altitudes (Shalimov and Gokhberg, 1998; Pulinets and Boyarchuk, 2004; Liperovsky et al., 2008). There, due to collisions between the neutral and charged particles, disturbances of the charged particle densities are possible. In ionospheric E-layers, atmospheric acous- tic and acoustic-gravity waves interact in particular with sporadic layers and cause nonlinear current systems and diffusion processes.

Liperovskaya et al. (1994) found that acoustic-gravity waves with periods of 2-3 h can apparently cause the diffusion of sporadic E-layers at distances of about $1000 \mathrm{~km}$ from the wave generation region. Further, upon investigating the interaction of infrasound waves of seismic origin with sporadic layers, it was found that waves observable by groundbased radar stations of the Farley-Buneman type, may be excited (Liperovsky et al., 1997; Meister, 1995).

Besides this, in (Koshevaya et al., 2002) it was concluded that at E-region altitudes, the conversion of sound waves into Alfvén waves is possible.

The theoretical description of the wave conversion in a stratified magnetized plasma has yet to be further developed. Solutions for acoustic waves are well-described for non-magnetic systems using one-fluid magnetohydrodynamics; when studying electromagnetic waves one usually neglects the stratification of the medium and the finite electrical conductivity values (Koshevaya et al., 2002; Sturrock, 1994) or the collisions between neutral and charged particles (Axelsson, 1998). Nonetheless it is of interest to analyse the modification of electromagnetic waves that have been locally excited in the atmosphere by acoustic-type ones. In the work of (Meister et al., 2010), some first steps were made to consider within the frame of multi-fluid magnetohydrodynamics, altitudinal profiles of particle velocities and electromagnetic fields in an isothermal system. Thus, in the present paper it is attempted to consider the modulation of magnetoacoustic waves by acoustic-type ones within the frame of multi-fluid magnetohydrodynamics, taking into account altitudinal profiles of the non-isothermal, isotropic ionospheric E-layer.

Correspondence to: $\mathrm{C} . \mathrm{-V}$. Meister

(c.v.meister@ skmail.ikp.phyik.tu-

darmstadt.de)

Published by Copernicus Publications on behalf of the European Geosciences Union. 


\section{Variation of electromagnetic waves by acoustic ones}

In the proposed multi-fluid magnetohydrodynamic model, the variation and excitation of electromagnetic waves by acoustic-type ones is studied starting with the continuity equations of the charged ( $a=\mathrm{e}-$ electron, $a=\mathrm{i}-\mathrm{ion}$ ), and neutral $(a=\mathrm{n})$ particles,

$$
\frac{\partial n_{a}}{\partial t}+\nabla\left(n_{a} \boldsymbol{v}_{a}\right)=0
$$

the momentum balances

$$
\begin{gathered}
n_{a} \frac{\partial \boldsymbol{v}_{a}}{\partial t}=-\frac{\operatorname{grad} p_{a}}{m_{a}}+\frac{q_{a} n_{a}}{m_{a}} \boldsymbol{E}+n_{a}\left[\boldsymbol{v}_{a} \times \boldsymbol{\omega}_{a}\right] \\
+n_{a} \boldsymbol{g}-\frac{n_{a}}{m_{a}} \sum_{b} m_{a b} v_{a b}\left(\boldsymbol{v}_{a}-\boldsymbol{v}_{b}\right)
\end{gathered}
$$

and the equation of state

$$
\begin{gathered}
{\left[\frac{\partial}{\partial t}+\boldsymbol{v} \nabla\right]\left(p \rho^{-\gamma}\right)=0, \quad p=\sum_{a} p_{a},} \\
\rho=\sum_{a} m_{a} n_{a}, \quad \rho \boldsymbol{v}=\sum_{a} m_{a} n_{a} \boldsymbol{v}_{a} .
\end{gathered}
$$

$\boldsymbol{v}_{a}, m_{a}, n_{a}, \boldsymbol{\omega}_{a}$, and $p_{a}$ describe the velocities, masses, number densities, cyclotron frequencies, and the partial pressure of the particles of type a, respectively. $m_{a b}=m_{a} m_{b} /\left(m_{a}+\right.$ $\left.m_{b}\right), \gamma$ is the polytropic coefficient, and $v_{a b}$ are the frequencies of the collisions between particles of kinds $a$ and $b . b$ designates charged and neutral particles too. Besides, the Maxwell equations are taken into account (replacement currents are neglected),

$$
\begin{gathered}
\operatorname{rot} \boldsymbol{B}(\boldsymbol{r}, t)=\mu_{o} \boldsymbol{j}(\boldsymbol{r}, t)=\mu_{o} q_{e}\left(n_{e} \boldsymbol{v}_{e}-n_{i} \boldsymbol{v}_{i}\right), \\
\operatorname{rot} \boldsymbol{E}(\boldsymbol{r}, t)=-\frac{\partial \boldsymbol{B}(\boldsymbol{r}, t)}{\partial t}, \\
\operatorname{div} \boldsymbol{B}(\boldsymbol{r}, t)=0 \\
\operatorname{div} \boldsymbol{E}(\boldsymbol{r}, t)=\frac{q_{e}}{\varepsilon_{o}}\left(n_{e}-n_{i}\right) .
\end{gathered}
$$

Under equilibrium conditions (index "o"), when all particle velocities equal zero, one obtains for the momentum balance

$$
-\frac{\operatorname{grad} p_{a o}}{m_{a}}+\frac{q_{a} n_{a o}}{m_{a}} \boldsymbol{E}_{o}+n_{a o} \boldsymbol{g}=0
$$

the solution

$$
\begin{aligned}
n_{a o}(z) & =n_{a o}\left(z_{o}\right) \frac{T_{o}\left(z_{o}\right)}{T_{o}(z)} \exp \left(-z / H_{a}(z)\right) . \\
H_{a}(z) & =z /\left\{\int_{z_{o}}^{z} \frac{m_{n} g-q_{a} E_{o z}\left(z^{*}\right)}{k_{B} T_{o}\left(z^{*}\right)} d z^{*}\right\}
\end{aligned}
$$

is the altitudinal-dependent scale height of the pressure of the Earth's atmosphere and ionosphere. Further, the background

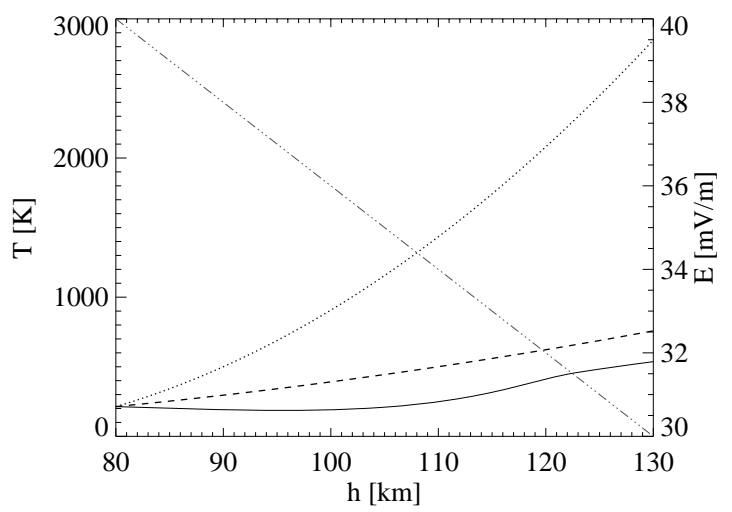

Fig. 1. Temperature according to the Mikhailov-model Eq. (12) (dashed line $-\alpha=0.1$, dotted line $-\alpha=0.3$ ), experimental MSISE-90 Atmosphere Model temperature data (VITMO) (full line), and present electric field model $E_{O z}=E_{o}(1+a z)$ (dash-dot-dot-dot line) in dependence on the altitude.

vertical electric field is approximated by a linear expression $E_{o z}(z)=E_{o}(z=0)(1+a z), a=-5 \times 10^{-3} \mathrm{~km}, E_{o}(z=0)$ $=40 \mathrm{mV} \mathrm{m}^{-1}$ is found fitting $E_{o z}(z)$ to data presented in (Pfaff et al., 2005). $z=0$ corresponds to an altitude of $80 \mathrm{~km}$. Values of the background electric field at altitudes between $80 \mathrm{~km}$ and $130 \mathrm{~km}$ are presented in Fig. 1. It has to be noted, that the direction of $\boldsymbol{E}$ considered in (Pfaff et al., 2005) is unclear. Here it is assumed that the presented data are those of the vertical electric field.

Further, taking into account that the height scale of the density of the oxygen molecules $\mathrm{O}_{2}$ with mass $m_{n}$ may be described by $H_{\mathrm{n}}(z)=H_{o}+\alpha z, \alpha=0.1-0.3, H_{o}=k_{B} T\left(z_{o}=\right.$ $0) /\left(m_{\mathrm{n}} g\right)$ (Michailov et al., 2007), one finds for the temperature $T$ of the E-layer, which is equal for all particles

$$
T(z)=\frac{m_{n} g\left(H_{o}+\alpha z\right)^{2}}{k_{B} H_{o}}, \quad \frac{\partial T(z)}{\partial z}=\frac{2 \alpha T(z)}{H_{o}+\alpha z} .
$$

Then for the altitudinal scale height of the pressure of neutral and ionized particles of mean mass $m_{n} \approx m_{i}$ and charge $q_{a}$ follows

$$
\begin{aligned}
& -\frac{z}{H_{a}(z)} \approx-H_{o} \int_{0}^{z} \frac{m_{n} g-q_{a} E_{o}(1+a z)}{m_{n} g\left(H_{o}+\alpha z\right)^{2}} d z=-\frac{z}{H_{o}+\alpha z} \\
& +\frac{q_{a} E_{o} z}{m_{n} g\left(H_{o}+\alpha z\right)}\left(1-\frac{H_{o} a}{\alpha}\right)+\frac{H_{o} q_{a} E_{o} a}{m_{n} g \alpha^{2}} \ln \left|\frac{\alpha z}{H_{o}}+1\right| .
\end{aligned}
$$

Temperature profiles of the E-layer according to Eq. (12) are presented in Fig. 1 for $\alpha$ equal to 0.1 and 0.3. The temperature values are compared with experimental data taken from the MSIS-E-90 Atmospheric Model of the Virtual Ionosphere, Thermosphere, Mesosphere Observatory (VITMO) (http://omniweb.gsfc.nasa.gov/vitmo/msis_vitmo.html). 
Next, it is supposed that the plasma system is perturbed by an acoustic-gravity or infrasound wave, expressed by the velocity of the neutral particles $\boldsymbol{v}_{n}$. In such a case, also the partial pressures and densities of the plasma particles as well as the electromagnetic field show deviations from the equilibrium values,

$$
\begin{gathered}
\boldsymbol{v}_{a}=\delta \boldsymbol{v}_{a}, \quad n_{a}=n_{a o}+\delta n_{a}, \quad p_{a}=p_{a o}+\delta p_{a}, \\
\boldsymbol{E}=\boldsymbol{E}_{o}+\delta \boldsymbol{E}, \quad \boldsymbol{B}=\boldsymbol{B}_{o}+\delta \boldsymbol{B} .
\end{gathered}
$$

The index " $o$ " designates the unperturbed values of the parameters. In the following it is assumed that the unperturbed electric and magnetic fields are directed along the $z$-axis. The mean electric field $\boldsymbol{E}_{o}$ is altitudinal dependent, and the mean magnetic field $\boldsymbol{B}_{o}$ is constant. Viscosity effects are neglected.

Substituting Eq. (14) into Eq. (8) and averaging over the deviations, one finds

$$
n_{\mathrm{e} o}(z)=n_{\mathrm{i} o}(z)+\eta, \quad \eta=\frac{\varepsilon_{o} a E_{o}}{q_{\mathrm{e}}} .
$$

Then the altitudinal profile of the background electron pressure may be presented by

$$
\begin{gathered}
p_{\mathrm{e} o}(z)=k_{B} n_{\mathrm{e} o}(z) T_{o}(z) \\
=k_{B} n_{\mathrm{i} o}(z) T_{o}(z)+\eta k_{B} T_{o}(z)=p_{\mathrm{i} o}(z)+\eta k_{B} T_{o}(z) \\
=p_{\mathrm{i} o}(z=0) \exp \left[-z / H_{i}(z)\right]+\eta k_{B} T_{o}(z) .
\end{gathered}
$$

Further, putting Eq. (14) into Eqs. (1-3), subtracting the equilibrium values, and retaining only terms of first order in the perturbations, one arrives at the following equations $\left(\boldsymbol{\omega}_{c a}=q_{a} \boldsymbol{B} / m_{a}\right)$ :

$$
\begin{gathered}
\frac{\partial \delta n_{a}}{\partial t}+\nabla\left(n_{a o} \delta \boldsymbol{v}_{a}\right)=0 \\
n_{a o} \frac{\partial \delta \boldsymbol{v}_{a}}{\partial t}=-\frac{1}{m_{a}} \operatorname{grad} \delta p_{a}+\frac{q_{a}}{m_{a}} \delta n_{a} \boldsymbol{E}_{o z}+\frac{q_{a}}{m_{a}} n_{a o} \delta \boldsymbol{E} \\
+n_{a o} \delta \boldsymbol{v}_{a} \times \boldsymbol{\omega}_{a o}+\delta n_{a} \boldsymbol{g}-\frac{n_{a o}}{m_{a}} \sum_{b} m_{a b} v_{a b}\left(\delta \boldsymbol{v}_{a}-\delta \boldsymbol{v}_{b}\right) \\
\delta \boldsymbol{v}_{a}\left[\rho \nabla p_{o}-\gamma p_{o} \nabla \rho_{o}\right]+\rho_{o} \frac{\partial \delta p}{\partial t}-\gamma p_{o} \frac{\partial \delta \rho}{\partial t}=0
\end{gathered}
$$

From the Maxwell equations follows for the electromagnetic field of the plasma disturbances

$$
\begin{gathered}
\operatorname{rot} \delta \boldsymbol{E}=-\frac{\partial \delta \boldsymbol{B}}{\partial t}, \\
\operatorname{div} \delta \boldsymbol{E}=\frac{q_{e}}{\varepsilon_{o}}\left(\delta n_{e}-\delta n_{i}\right), \\
\operatorname{rot} \delta \boldsymbol{B}=\mu_{o} \delta \boldsymbol{j}=\mu_{o} q_{e}\left(n_{\mathrm{e} o} \delta \boldsymbol{v}_{e}-n_{\mathrm{i} o} \delta \boldsymbol{v}_{i}\right),
\end{gathered}
$$

$$
\operatorname{div} \delta \boldsymbol{B}=0 .
$$

Usually, to obtain the relations for the waves excited in the non-stratified plasma, one introduces the Fourier transformation of the plasma parameters and of the electromagnetic field. But, since the background plasma pressure and particle densities show an exponential decrease with the altitude, solving the system of Eqs. (17-23), for the plasma and electromagnetic field parameters here the following expressions are assumed:

$$
\begin{gathered}
\delta Z=\delta Z_{o} \exp \left\{-\frac{z}{2 H_{a}}+i \boldsymbol{k} \boldsymbol{r}-i \omega t\right\}, \\
Z=\left\{n_{a}, p_{a}, E_{\mathrm{z}}, B_{\mathrm{z}}\right\}, \quad a=\{i, n\}, \\
\delta Z=\delta Z_{o} \exp \left\{-\frac{z}{2 H_{i}}+i \boldsymbol{k} \boldsymbol{r}-i \omega t\right\}, \\
Z=\left\{E_{i}, B_{i}\right\}, \quad i=\{x, y\}, \\
\delta \boldsymbol{v}_{a}=\delta \boldsymbol{v}_{a o} \exp \left\{\frac{z}{2 H_{a}}+i \boldsymbol{k r}-i \omega t\right\},
\end{gathered}
$$

It is of importance that, omitting horizontal profiles of the electromagnetic field, the altitudinal scales of this field $\left(H_{\mathrm{x}}\right.$ and $H_{\mathrm{y}}$ ) have to be chosen differently in the $\mathrm{x}$ - and $\mathrm{y}$ directions. Otherwise, no magnetic field disturbances will occur. Thus, the model presented here is valid only locally, and there have to be experimental data used for the horizontal scales.

Substituting Eq. (24) in the continuity Eq. (17), one gets $(a=i, n)$

$$
\begin{gathered}
i \omega \delta n_{a o}=\frac{p_{a o}}{k_{B} T_{o}}\left(i \boldsymbol{k}-u \boldsymbol{n}_{\mathrm{z}}\right) \delta \boldsymbol{v}_{a o}-\frac{2 p_{a o}}{k_{B} T_{o}}\left(u+\frac{\alpha}{H_{o}+\alpha z}\right) \delta v_{a o z}, \\
i \omega \delta n_{\mathrm{e} o}=\frac{p_{\mathrm{i} o}+\eta}{k_{B} T_{o}}\left(i \boldsymbol{k}-u \boldsymbol{n}_{\mathrm{z}}\right) \delta \boldsymbol{v}_{\mathrm{e} o}-\frac{2 p_{\mathrm{i} o}}{k_{B} T_{o}}\left(u+\frac{\alpha}{H_{o}+\alpha z}\right) \delta v_{\mathrm{e} o z}, \\
u=\frac{1}{2 H_{i}}-\frac{z}{2 H_{i}^{2}} \frac{\partial H_{i}}{\partial z} .
\end{gathered}
$$

The index $i$ in Eq. (29) describes the ions. For the linearized equation of state Eq. (19) follows

$$
\begin{gathered}
-i \omega \delta p_{o}+i \gamma \frac{p_{o} \omega}{\rho_{o}} \delta \rho_{o}+\delta v_{o z} s(z)=0, \\
\delta p_{o}=\sum_{a} \delta p_{a o}, \delta \rho_{o}=\sum_{a} m_{a} \delta n_{a o}, \\
\delta \boldsymbol{v}_{o}=\sum_{a} m_{a} n_{a o} \delta \boldsymbol{v}_{a o} / \rho_{o}, \\
s(z)=-2 u\left(p_{n o}+2 p_{\mathrm{i} o}\right)+\frac{2 \eta k_{B} \alpha T_{o}(z)}{\left(H_{o}+\alpha z\right)} \\
+\frac{2 \gamma p_{o}}{\rho_{o}} \frac{\left(p_{n o} m_{n}+p_{\mathrm{i} o} m_{i}\right)}{k_{B} T_{o}}\left[u+\frac{\alpha}{H_{o}+\alpha z}\right] .
\end{gathered}
$$

Nat. Hazards Earth Syst. Sci., 11, 1011-1017, 2011 
Expressing the plasma pressure $p$ by the equation of state of the ideal plasma, $p_{a}=k_{B} T n_{a}$, one obtains with

$$
\delta p_{a}=k_{B} T_{o} \delta n_{a}+n_{a o} k_{B} \delta T
$$

from Eq. (30) for the temperature fluctuations in the E-layer

$$
\delta T=\frac{\gamma p_{o} \omega / \rho_{o} \delta \rho-\omega k_{B} T \sum_{a} \delta n_{a}-i s(z) \delta v_{z}}{\omega k_{B} \sum_{a} n_{a o}} .
$$

Further, the linearized Maxwell equations Eqs. (20-22) may be transformed into the relations

$$
\begin{aligned}
& \delta E_{o x} e^{z /\left(2 H_{i}\right)-z /\left(2 H_{\mathrm{x}}\right)}=\frac{i \omega \mu_{o} q_{e}}{k_{\mathrm{x}}^{2} k_{\mathrm{y}}^{2}\left(u_{\mathrm{x}}-u_{\mathrm{y}}\right)^{2}} \\
& \left\{\left(n_{\mathrm{e} o} \delta v_{\mathrm{e} o x}-n_{\mathrm{i} o} \delta v_{i o x}\right) k_{\mathrm{x}}^{2}\left[k_{\perp}^{2}+\left(k_{\|}+i u_{\mathrm{y}}\right)\left(k_{\|}+i u_{\mathrm{x}}\right)\right]\right. \\
& +\left(n_{\mathrm{e} o} \delta v_{\mathrm{e} o y}-n_{\mathrm{i} o} \delta v_{i o y}\right)\left[k_{\perp}^{2}+\left(k_{\|}+i u_{\mathrm{y}}\right)^{2}\right] k_{\mathrm{x}} k_{\mathrm{y}} \\
& +\left(n_{\mathrm{e} o} \delta v_{\mathrm{e} o z}-n_{\mathrm{i} o} \delta v_{i o z}\right)\left[k_{\mathrm{x}}\left(k_{\|}+i u_{\mathrm{y}}\right)^{2}\left(k_{\|}+i u_{\mathrm{x}}\right)\right. \\
& \left.\left.+k_{\mathrm{y}}^{2} k_{\mathrm{x}}\left(k_{\|}+i u_{\mathrm{x}}\right)+k_{\mathrm{x}}^{3}\left(k_{\|}+i u_{\mathrm{y}}\right)\right]\right\} \\
& \delta E_{o y} e^{z /\left(2 H_{i}\right)-z /\left(2 H_{\mathrm{y}}\right)}=\frac{i \omega \mu_{o} q_{e}}{k_{\mathrm{x}}^{2} k_{\mathrm{y}}^{2}\left(u_{\mathrm{x}}-u_{\mathrm{y}}\right)^{2}} \\
& \left\{\left(n_{\mathrm{e} o} \delta v_{\mathrm{e} o x}-n_{\mathrm{i} o} \delta v_{i o x}\right)\left[k_{\perp}^{2}+\left(k_{\|}+i u_{\mathrm{x}}\right)^{2}\right] k_{\mathrm{x}} k_{\mathrm{y}}\right. \\
& +\left(n_{\mathrm{e} o} \delta v_{\mathrm{e} o y}-n_{\mathrm{i} o} \delta v_{i o y}\right) k_{\mathrm{y}}^{2}\left[k_{\perp}^{2}+\left(k_{\|}+i u_{\mathrm{x}}\right)\left(k_{\|}+i u_{\mathrm{y}}\right)\right] \\
& +\left(n_{\mathrm{e} o} \delta v_{\mathrm{e} o z}-n_{\mathrm{i} o} \delta v_{i o z}\right)\left[k_{\mathrm{y}}\left(k_{\|}+i u_{\mathrm{x}}\right)^{2}\left(k_{\|}+i u_{\mathrm{y}}\right)\right. \\
& \left.\left.+k_{\mathrm{x}}^{2} k_{\mathrm{y}}\left(k_{\|}+i u_{\mathrm{y}}\right)+k_{\mathrm{y}}^{3}\left(k_{\|}+i u_{\mathrm{x}}\right)\right]\right\} \\
& \delta E_{o z}=\frac{i \omega \mu_{o} q_{e}}{k_{\mathrm{x}}^{2} k_{\mathrm{y}}^{2}\left(u_{\mathrm{x}}-u_{\mathrm{y}}\right)^{2}} \\
& \left\{( n _ { \mathrm { e } o } \delta v _ { \mathrm { e } o x } - n _ { \mathrm { i } o } \delta v _ { i o x } ) k _ { \mathrm { x } } \left[k_{\mathrm{x}}^{2}\left(k_{\|}+i u_{\mathrm{X}}\right)\right.\right. \\
& \left.+k_{\mathrm{y}}^{2}\left(k_{\|}+i u_{\mathrm{y}}\right)+\left(k_{\|}+i u_{\mathrm{x}}\right)^{2}\left(k_{\|}+i u_{\mathrm{y}}\right)\right] \\
& +\left(n_{\mathrm{e} o} \delta v_{\mathrm{e} o y}-n_{\mathrm{i} o} \delta v_{i o y}\right) k_{\mathrm{y}}\left[k_{\mathrm{y}}^{2}\left(k_{\|}+i u_{\mathrm{y}}\right)\right. \\
& \left.+k_{\mathrm{x}}^{2}\left(k_{\|}+i u_{\mathrm{x}}\right)+\left(k_{\|}+i u_{\mathrm{x}}\right)\left(k_{\|}+i u_{\mathrm{y}}\right)^{2}\right] \\
& +\left(n_{\mathrm{e} o} \delta v_{\mathrm{e} o z}-n_{\mathrm{i} o} \delta v_{i o z}\right)\left[k_{\perp}^{2}\left(k_{\|}+i u_{\mathrm{x}}\right)\left(k_{\|}+i u_{\mathrm{y}}\right)\right. \\
& \left.\left.+\left(k_{\|}+i u_{\mathrm{x}}\right)^{2}\left(k_{\|}+i u_{\mathrm{y}}\right)^{2}\right]\right\}, \\
& u_{\mathrm{x}}=\frac{1}{2 H_{\mathrm{x}}}-\frac{z}{2 H_{\mathrm{x}}^{2}} \frac{\partial H_{\mathrm{x}}}{\partial z}, \quad u_{\mathrm{y}}=\frac{1}{2 H_{\mathrm{y}}}-\frac{z}{2 H_{\mathrm{y}}^{2}} \frac{\partial H_{\mathrm{y}}}{\partial z} .
\end{aligned}
$$

From the linearized momentum equation Eq. (18) follows

$$
\begin{aligned}
& -i \omega \delta \boldsymbol{v}_{n o}=-\frac{\left(i \boldsymbol{k}-u \boldsymbol{n}_{\mathrm{z}}\right) \delta p_{n o}}{m_{n} n_{n o}}+\boldsymbol{g} \delta n_{n o} / n_{n o} \\
& -\frac{m_{i} v_{n i}}{m_{i}+m_{n}}\left(\delta \boldsymbol{v}_{n o}-\delta \boldsymbol{v}_{\mathrm{i} o}\right)-\frac{m_{e} v_{n e}}{m_{n}}\left(\delta \boldsymbol{v}_{n o}-\delta \boldsymbol{v}_{\mathrm{e} o}\right), \\
& -i \omega \delta \boldsymbol{v}_{\mathrm{e} o}=-\frac{\left(i \boldsymbol{k}-u \boldsymbol{n}_{\mathrm{z}}\right) \delta p_{\mathrm{e} o}}{m_{e} n_{\mathrm{e} o}}+\frac{q_{e} E_{o z} \boldsymbol{n}_{\mathrm{z}}}{m_{e} n_{\mathrm{e} o}} \delta n_{\mathrm{e} o} \\
& +\frac{q_{e}}{m_{e}} e^{-z / 2 H_{i}(z)-i \boldsymbol{k} \boldsymbol{r}+i \omega t} \delta \boldsymbol{E}+\omega_{c e} \delta v_{\mathrm{e} o y} \boldsymbol{n}_{\mathrm{x}}-\omega_{c e} \delta v_{\mathrm{e} o x} \boldsymbol{n}_{\mathrm{y}} \\
& +\boldsymbol{g} \delta n_{\mathrm{e} o} / n_{\mathrm{e} o}-v_{\mathrm{e} i}\left(\delta \boldsymbol{v}_{\mathrm{e} o}-\delta \boldsymbol{v}_{\mathrm{i} o}\right)-v_{\mathrm{e} n}\left(\delta \boldsymbol{v}_{\mathrm{e} o}-\delta \boldsymbol{v}_{n o}\right), \\
& -i \omega \delta \boldsymbol{v}_{\mathrm{i} o}=-\frac{\left(i \boldsymbol{k}-u \boldsymbol{n}_{\mathrm{z}}\right) \delta p_{\mathrm{i} o}}{m_{i} n_{\mathrm{i} o}}+\frac{q_{i} E_{o} \boldsymbol{n}_{\mathrm{z}}}{m_{i} n_{\mathrm{i} o}} \delta n_{\mathrm{i} o} \\
& +\frac{q_{i}}{m_{i}} e^{-z / 2 H_{i}(z)-i \boldsymbol{k} \boldsymbol{r}+i \omega t} \delta \boldsymbol{E} \\
& +\omega_{c i} \delta v_{i o y} \boldsymbol{n}_{\mathrm{x}}-\omega_{c i} \delta v_{i o x} \boldsymbol{n}_{\mathrm{y}}+\boldsymbol{g} \delta n_{\mathrm{i} o} / n_{\mathrm{i} o} \\
& -v_{i e} \frac{m_{e}}{m_{i}}\left(\delta \boldsymbol{v}_{\mathrm{i} o}-\delta \boldsymbol{v}_{\mathrm{e} o}\right)-v_{i n} \frac{m_{n}}{m_{n}+m_{i}}\left(\delta \boldsymbol{v}_{\mathrm{i} o}-\delta \boldsymbol{v}_{n o}\right) \text {. }
\end{aligned}
$$

Thus, expressing in the equations Eqs. (38-40) $\delta p_{a o}$ by the formulae Eqs. $(33,34), \delta \boldsymbol{E}_{o}$ by Eqs. (35-7), as well as $\delta n_{a o}$ and $\delta n_{\mathrm{e} o}$ by Eqs. (27-29), one finds a dispersion relation of magnetohydrodynamic waves in the ionospheric E-layer caused by the motion of neutral and ionized particles. The expression for the dispersion relation is rather large. Thus it will not be given here. It has to be solved numerically. Here, only a first discussions of the special solution of Alfvén waves modified by the acoustic ones is presented.

If one forms the sum of Eqs. $(39,40)$ describing the momentum balance of the plasma components of the system, one obtains $\left(n_{\mathrm{e} o} v_{\mathrm{e} i}=n_{\mathrm{i} o} v_{i e}, m_{i} \approx m_{n}\right)$

$$
\begin{gathered}
-i \omega \rho_{o} \delta \boldsymbol{v}_{1 o}=-\left(i \boldsymbol{k}-u \boldsymbol{n}_{\mathrm{Z}}\right) \delta p_{o} \\
-e E_{o z} \boldsymbol{n}_{\mathrm{Z}}\left(\delta n_{\mathrm{e} o}-\delta n_{\mathrm{i} o}\right)-e \eta e^{\frac{-z}{2 H_{i}}-i \boldsymbol{k} \boldsymbol{r}+i \omega t} \delta \boldsymbol{E} \\
+\frac{1}{\mu_{o}} e^{\frac{-z}{2 H_{a}}-i \boldsymbol{k} \boldsymbol{r}+i \omega t}\left[\operatorname{rot} \delta \boldsymbol{B} \times \boldsymbol{B}_{o}\right]+\boldsymbol{g}\left(m_{e} \delta n_{\mathrm{e} o}+m_{i} \delta n_{\mathrm{i} o}\right) \\
-v_{\mathrm{e} n} m_{e} n_{\mathrm{e} o}\left(\delta \boldsymbol{v}_{\mathrm{e} o}-\delta \boldsymbol{v}_{n o}\right)-v_{i n} \frac{m_{i} n_{\mathrm{i} o}}{2}\left(\delta \boldsymbol{v}_{\mathrm{i} o}-\delta \boldsymbol{v}_{n o}\right) . \\
\delta \boldsymbol{v}_{1 o}=\sum_{a=e, i} m_{a} n_{a o} \delta \boldsymbol{v}_{a o} /\left(m_{e} n_{\mathrm{e} o}+m_{i} n_{\mathrm{i} o}\right) .
\end{gathered}
$$

The $y$-component of this relation reads

$$
\begin{gathered}
-i \omega \rho_{o} \delta v_{1 o y}=-i k_{\mathrm{y}} \delta p_{o}+e \eta e^{\frac{-z}{2 H_{i}}-i \boldsymbol{k r}+i \omega t} \delta E_{y} \\
+\frac{1}{\mu_{o}} B_{o z}\left(i k_{\mathrm{y}} \delta B_{o z}+\left[u_{\mathrm{y}}-i k_{\|}\right] e^{\frac{-z}{2 H_{i}}-i \boldsymbol{k r}+i \omega t} \delta B_{y}\right) \\
-v_{\mathrm{e} n} m_{e} n_{\mathrm{e} o}\left(\delta v_{\mathrm{e} o y}-\delta v_{n o y}\right)-v_{i n} \frac{m_{i} n_{\mathrm{i} o}}{2}\left(\delta v_{i o y}-\delta v_{n o y}\right)
\end{gathered}
$$


If one neglects particle collisions, the electric background field (considering infinite electrical conductivity values) and the stratification of the atmosphere, the $y$ component of Eq. (42) coincides with the dispersion relation of Alfvén waves usually considered (see, e.g. Sturrock, 1994, Eqs. 14.1.25, 14.1.29). For wave vectors in the x-Zplane (this assumption does not restrict the general solution), $\delta v_{o y}$ is proportional to $\delta B_{o y}$. One finds a dispersion relation

$$
\omega^{2}=v_{A}^{2} k_{\|}^{2}, \quad v_{A}^{2}=\frac{B_{o}^{2}}{m u_{o}\left(n_{\mathrm{e} o} m_{e}+n_{\mathrm{i} o} m_{i}\right)},
$$

for the waves which are transverse to the background magnetic field.

Collisions of charged particles with the neutral ones change the growth rate of the Alfvén waves, as follows from Eq. (42). In case of fluctuations of the velocities of the neutral particles larger than the fluctuations of the speeds of the charged particles $\left(\delta v_{\text {noy }}>\delta v_{\mathrm{e} o y}, \delta v_{\text {ioy }}\right)$, for instance by infrasound or acoustic-gravity waves, the wave amplitudes should grow. Further, taking into account a finite electrical conductivity of the atmosphere, $\delta v_{o y}$ is also correlated to magnetic field fluctuations $\delta B_{o z}$ parallel to the background field $\boldsymbol{B}_{o}$ as

$$
\delta E_{y}=\frac{i \omega}{k_{\mathrm{x}}\left(u_{\mathrm{y}}-u_{\mathrm{x}}\right)}\left[k_{\mathrm{x}} \delta B_{x}+k_{\mathrm{y}} \delta B_{y}+\left(k_{\|}+i u_{\mathrm{x}}\right) \delta B_{z}\right] .
$$

Thus, we have found that in the ionospheric E-layer, normal Alfvén waves may be generated. The amplitudes and propagation directions of the waves are modified by upstreaming acoustic waves. This phenomenon is possible when the frequency of the acoustic waves is larger than the cut-off frequency $\Omega=c_{s} / 2 H_{i}$, so that the waves penetrate into the E-layer (Liperovsky et al., 1997; Meister, 1995; Koshevaya et al., 2002). Via collisions of the neutral particles with the charged ones, the momentum of the neutral particles is transferred to the plasma components. Then the variations of the velocities of the charged particles generate an electromagnetic wave field. The waves may be Alfvén ones, but magnetoacoustic waves or the so-called Farley-Buneman waves may also occur. The wave generation is influenced by the background electromagnetic field and by the altitudinal profiles of the plasma parameters.

In Liperovsky et al. (1997), considering acoustic waves with frequencies of $5 \times 10^{-3}-20 \mathrm{~Hz}$ and neglecting viscosity effects, the Farley-Buneman waves were found to be caused by the $y$-components of the electron velocities. Neglecting altitudinal profiles of the plasma and magnetic wave fields $\delta \mathbf{B}$ in the E-layer, it was already found by (Meister, 1995) that always three electrostatic waves of the Farley-Buneman type (described by Eqs. 39, 40) exist. One wave possesses a growing amplitude, and the other two waves are damped. The non-damped wave has in the E-layer $\left(T=250 \mathrm{~K}, v_{\mathrm{en}}=\right.$ $4 \times 10^{4} \mathrm{~Hz}, v_{\mathrm{en}}=1.4 \times 10^{3} \mathrm{~Hz}, \omega_{c \mathrm{e}}=5.3 \times 10^{6} \mathrm{~Hz}$ ) wave numbers of the order of $1-70 \mathrm{~m}^{-1}$ and phase velocities of about $500 \mathrm{~ms}^{-1}$. The Farley-Buneman waves are driven by

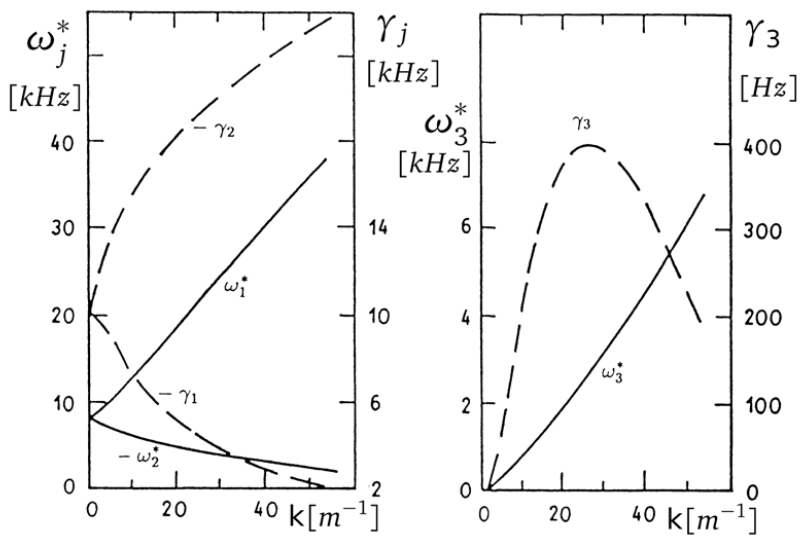

Fig. 2. Three solutions of the dispersion relation of FarleyBuneman waves. The wave frequencies equal $\omega_{j}=\omega_{j}^{*}+k_{\mathrm{y}} V_{\mathrm{eyo}}, \gamma_{i}$ describes the growth rates, $V_{\text {eyo }}$ - the amplitude of the electron Hall velocity exciting the waves, and $k$ is the wave number. $j=\{1,2,3\}$ (Meister, 1995).

the Hall velocity of the electrons, which is proportional to the neutral gas velocity, $v_{\mathrm{e} y} \approx 3-6 v_{n}$. The waves are excited under the condition that

$$
\begin{gathered}
v_{\mathrm{ey}}>V_{\text {crit }} \approx c_{s}(1+R), \\
c_{s}^{2}=\frac{\gamma p}{n_{\mathrm{e} o} m_{e}+n_{\mathrm{io}} m_{i}+n_{n o} m_{n}}, \quad R=\frac{-v_{\mathrm{en}} \nu_{\mathrm{in}}}{\omega_{e} \omega_{i}} .
\end{gathered}
$$

The Hall currents cause fluctuations of the magnetic field in the directions of the neutral gas motion and along the mean magnetic field.

The electron velocities parallel to the earth's magnetic field are of the order of $0.025 v_{\mathrm{e} z} a \approx 2.5-5 v_{\mathrm{e} y}$, where $a$ is the horizontal dimension of the sporadic E-layer amounting to a few hundreds of meters. Thus $v_{\mathrm{e} z}$ may reach 720 $1900 \mathrm{~ms}^{-1}$ at neutral gas velocities larger than $50 \mathrm{~ms}^{-1}$, and it may generate rather strong variations of the magnetic field. In addition, the plasma will be heated (Liperovsky and Meister, 1996).

\section{Discussion of the results and conclusions}

1. First steps have been performed to investigate the modification of the electromagnetic field of the ionospheric E-layer by acoustic-type waves, which might be generated by earthquake precursors or for meteorological reasons.

2. Using many-fluid magnetohydrodynamics, a full system of equations describing electromagnetic waves in the E-layer has been derived analytically. Influences of the background electromagnetic field as well as the stratification of the atmosphere have been taken into account. 
3. Concerning electromagnetic waves in the E-layer, one may conclude that usual (according to the work of Sturrock, 1994) magnetohydrodynamic waves like Alfvén and magnetoacoustic ones are generated in the E-layer. These waves are modified with respect to amplitude and the direction of motion by acoustic-type waves propagating into the E-layer from lower altitudes. Such acoustic-type waves might be of seismic origin. But they also occur for meteorological reasons.

4. Almost electrostatic waves of the Farley-Buneman type might also be generated in seismo-active regions at Elayer altitudes a few days before earthquakes. Solutions for the dispersion relation and excitation conditions of the waves are discussed.

5. Wave turbulence in the ionospheric E-layer, and especially in sporadic E-layers, should influence the ionograms observed by ionospheric vertical radar stations. And indeed, some tendencies of variations of Es-spread effects have been observed (Liperovsky et al., 1999a, b, 2000, 2005).

6. Additional Joule heating caused by acoustic waves and the corresponding increased intensities of the vertical atmospheric currents - result also in modifications of the charged particle densities of the ionosphere (Meister, 1995; Liperovsky and Meister, 1996; Liperovsky et al., 1996; Meister et al., 1998). Thus, changes in the characteristic frequencies foE might be obtained.

7. The complete numerical solution of the system of Eqs. $(27,28,32-37)$ is a task for future work.

Acknowledgements. The authors kindly thank P.-F. Biagi and another anonymous referee for constructive comments. They also thank E. V. Liperovskaya for valuable discussions and the copy-editor for the improvement of the English text.

Edited by: T. Maggipinto

Reviewed by: P. F. Biagi and J. Blecki

\section{References}

Axelsson, P.: Dispersion relations for waves in anisotropic and stratified magnetoplasmas, Phys. Scripta, 57, 242-245, 1998.

Cook, R. K.: Infrasound radiated during the Montana earthquake of 1959 August 18, Geophys. J. R. astr. Soc., 26, 191-198, 1971.

Hayakawa, M.: On the fluctuation spectra of seismoelectromagnetic phenomena, Nat. Hazards Earth Syst. Sci., 11, 301-308, doi:10.5194/nhess-11-301-2011, 2011.

Koshevaya, S. V., Grimalsky, V. V., Burlak, G. N., and Kotsarenko, A. N.: Acoustic channel of the lithosphere-ionosphere coupling, Ukr. J. Phys., 47 (2), 142-146, 2002.
Liperovskaya, E. V., Hristakis, N., Liperovsky, V. A., and Oleinik, M. A.: Effects of seismic and anthropogenic activity in the nighttime sporadic ionospheric E layer, Geomagn. Aeronomy, 34 (3), 56-59, 1994.

Liperovskaya, E. V., Silina, A. S., Saidshoev, A., Liperovsky, V. A., Meister, C.-V., and Vasil'eva, N. E.: On the $E_{S}$-spread effect of night-time sporadic layers, Geomagn. Aeronomy, 40 (1), 120122, 2000.

Liperovsky, V. A. and Meister, C.-V.: Influence of collisional heating on the current generation in moving mid-latitude sporadic E-layers, Adv. Space Res., 18 (3), 93-97, 1996.

Liperovsky, V. A., Meister, C.-V., Senchenkov, S. A., Popov, K. V., Oleynik, M. A., and Liperovskaya, E. V.: Consequences of current generation in the ionosphere caused by neutral wind action on $E_{S}$-clouds, J. Radiophysics, 39 (2), 241-249, 1996.

Liperovsky, V. A., Meister, C.-V., Schlegel, K., and Haldoupis, C.: Currents and turbulence in and near mid-latitude sporadic Elayers caused by strong acoustic impulses, Ann. Geophys., 15, 767-773, doi:10.1007/s00585-997-0767-x, 1997.

Liperovsky, V. A., Popov, K. V., Pokhotelov, O. A., Meister, C.V., Liperovskaya, E. V., and Alimov, O. A.: Ionospheric $f_{b} E_{S}$ frequency variations with time in a seismically active region, Izvestiya, Phys. Solid Earth, 35 (12), 1043-1048, 1999a.

Liperovsky, V. A., Senchenkov, S. A., Liperovskaya, E. V., Meister, C.-V., Roubtzov, L. N., and Alimov, O. A.: fbEs-frequency variations with scales of minutes in mid-latitude sporadic layers, Geomagn. Aeronomy, 39 (1), 131-134, 1999b.

Liperovsky, V. A., Meister, C.-V., Liperovskaya, E. V., Vasil'eva, N. E., and Alimov, O.: On spread-Es effects in the ionosphere before earthquakes, Nat. Hazards Earth Syst. Sci., 5, 59-62, doi:10.5194/nhess-5-59-2005, 2005.

Liperovsky, V. A., Pokhotelov, O. A., Meister, C.-V., and Liperovskaya, E. V.: Physical models of coupling in the lithosphereatmosphere-ionosphere system before earthquakes, Geomagn. Aeronomy, 48 (6), 795-806, 2008.

Meister, C.-V.: On the physical theory of plasmas in nonequilibrium, post-doctoral thesis, University Rostock, 178-181, 1995.

Meister, C.-V., Liperovsky, V. A., and Senchenkov, S. A.: Ionacoustic instability caused by neutral wind action on sporadic E-layers, Adv. Space Res., 21 (6), 911-914, 1998.

Meister, C.-V., Hoffmann, D. H. H., and Liperovsky, V. A.: Acoustic-gravity model of the lithosphere-atmosphereionosphere coupling before earthquakes, 5th Int. Conf. "Solarterrestrial links and physics of earthquake precursors", session 5: "Physics of Earthquake Precursors", Paratunka/Kamchatka, 2.-7. August 2010, Proceedings, 141-145, 2010.

Mikhailov, A. V., Depuev, V. H., and Depueva, A. H.: Synchronous $N \mathrm{mF} 2$ and $N \mathrm{mE}$ daytime variations as a key to the mechanism of quiet-time F2-layer disturbances, Ann. Geophys., 25, 483-493, doi:10.5194/angeo-25-483-2007, 2007.

Pfaff, R., Freudenreich, H., Yokoyama, T., Yamamoto, M., Fukao, S., Mori, H., Ohtsuki, S., and Iwagami, N.: Electric field measurements of DC and long wavelength structures associated with sporadic- $E$ layers and QP radar echoes, Ann. Geophys., 23, 2319-2334, doi:10.5194/angeo-23-2319-2005, 2005. 
Pulinets, S. A. and Boyarchuk, K. A.: Ionospheric precursors of earthquakes, Springer, Berlin, Heidelberg, New York, 49-88, 2004.

Rozhnoi, A., Solovieva, M., Molchanov, O., Biagi, P.-F., and Hayakawa, M.: Observation evidences of atmospheric Gravity Waves induced by seismic activity from analysis of subionospheric LF signal spectra, Nat. Hazards Earth Syst. Sci., 7, 625628, doi:10.5194/nhess-7-625-2007, 2007.
Shalimov, S. L. and Gokhberg, M. B.: Lithosphere-ionosphere coupling mechanism and its application in the case of the June 20, 1990 earthquake in Iran, J. Earthquake Predict. Res., 7, 98-111, 1998.

Sturrock, P. A.: Plasma physics. An introduction to the theory of astrophysical, geophysical, and laboratory plasmas, Cambridge University Press, New York, 233-246, 1994. 